\title{
Pensamiento computacional en las prácticas intelectuales del estudiante de educación básica
}

\author{
Computational thinking in the intellectual practices of the basic education \\ student
}

- MARIANA FERNÁNDEZ

mfernandezreina@gmail.com

Universidad del Zulia, Venezuela
- NORAILITH POLANCO

norailithp@gmail.com

Universidad del Zulia, Venezuela

Artículo recibido en septiembre 2018 / Arbitrado en octubre 2018 / Publicado en enero 2019

Resumen El tema central del trabajo se refirió a un tipo de pensamiento, el "pensamiento computacional", que puede ser adoptado por todos en pro de favorecer las propias competencias intelectuales y proceder a la solución de problemas complejos de manera eficiente, de allí que el objetivo de este artículo fue reflexionar sobre la importancia de este tipo de pensamiento en las prácticas intelectuales de los estudiantes de educación básica, para finalmente sugerir algunas estrategias dirigidas a su desarrollo. Se caracterizó como una investigación descriptiva con diseño documental - bibliográfico. El principal resultado dio manifestaciones de las tendencias en la comunicación electrónica aplicada a procesos en educación, con las que se pueden obtener interpretaciones válidas para la adaptación curricular en primera etapa y su proyección para las generaciones modernas. Se concluye que el pensamiento computacional constituye una competencia esencial para el avance de las prácticas intelectuales de los educandos, con mayor provecho durante las etapas iniciales del desarrollo humano.

Palabras clave: Pensamiento computacional; estudiante; educación básica

\begin{abstract}
The central theme of the work referred to a type of thinking, "computational thinking", which can be adopted by all in favor of promoting their own intellectual competences and proceeding to solve complex problems efficiently, hence the objective of this article was to reflect on the importance of this type of thinking in the intellectual practices of basic education students, to finally suggest some strategies aimed at their development. It was characterized as a descriptive investigation with a documentary-bibliographic design. The main result gave manifestations of trends in electronic communication applied to processes in education, with which valid interpretations can be obtained for curricular adaptation in the first stage and its projection for modern generations. It is concluded that computational thinking constitutes an essential competence for the advancement of the intellectual practices of the learners, with greater benefit during the initial stages of human development.
\end{abstract}

Keywords: Computational thinking; student; basic education 


\section{INTRODUCCIÓN}

El tema de interés de esta investigación fue el "pensamiento computacional" y se refiere éste a un tipo de pensamiento, una idea, que conlleva a un hábito personal que puede ser adoptado por todos, en pro de favorecer las competencias intelectuales y capacita a quien lo aplique, para proceder a la solución de problemas complejos de una manera eficiente.

Como punto introductorio, es necesario reconocer que hoy día las personas conviven rodeadas de un amplio abanico de posibilidades adaptativas en variados aspectos sociales, por lo que haciendo honor al modernismo, los profesionales de cualquier disciplina deben ir de la mano con los consorcios cambiantes y trascendentales actuales denominados sociedades del conocimiento, para lo que se requiere someter el llamado status quo a constantes revisiones, con proyección a una premisa de incesante mejoramiento.

El agigantado auge tecnológico en el que se desenvuelven las actuales sociedades, ha permitido exponer la forma en la que los escenarios económicos en cualquier locación del globo, requieren hoy más que nunca de personas cualificadas en el tratamiento de datos e información automatizada, indistintamente del perfil profesional $u$ orientación laboral, debido a que la modernidad demanda $-y$ lo seguirá haciendo- que los quehaceres asociados a los oficios en cualquier sector productivo, sean migrados a sistemas informáticos, para su sostenibilidad, agilidad y trascendencia en el tiempo.

Con base en lo anterior, el objetivo de este estudio fue reflexionar sobre la importancia del pensamiento computacional en las prácticas intelectuales de los estudiantes de educación básica, y finalmente sugerir algunas estrategias dirigidas a su desarrollo, considerado éste desde su incorporación en diseños curriculares basados en experiencias formativas que contemplen estrategias para el desarrollo de distintas maneras de pensar, como competencia cardinal para los niños de educación básica, por ser un método complementario a la capacidad analítica del infante.

En este sentido, el presente trabajo se enfocó en revisar los aspectos teóricos que han sido aportados por expertos en el tema, sistematiza términos que aluden a las ciencias informáticas $\mathrm{O}$ procesos de programación de sistemas, conjuntamente con su instalación y repercusión dentro de los planes de estudio en educación de etapa básica.

De igual manera, se plasmaron ciertas ideas que pudiesen ser orientadas estratégicamente en los procesos de aprendizaje en las aulas de primaria, a fin de seguir contribuyendo con la comunidad de educadores, fundaciones, empresas e instituciones, quienes, en tiempos recientes, precisan la incorporación de este tipo de pensamiento en las prácticas formativas desde edades tempranas.

Asimismo, se presenta a continuación la conceptualización, características, elementos, pasos e importancia que tiene este tipo de pensamiento en el individuo, específicamente su aplicación en la infancia, cuando se convierte en punto clave de los procesos de resolución lógica de faenas diarias. De igual manera se considera el 
término pensamiento computacional dentro del plano educativo, mediante reflexiones sobre su incorporación en los diseños curriculares, para finalmente proponer algunas estrategias para su desarrollo.

Finalmente, se argumentan opiniones conclusivas en torno a los conceptos estudiados, que extienden las propias reflexiones y posturas teóricas.

Para esta reflexión se valoró un conjunto de referentes de la trayectoria del término. El concepto de pensamiento computacional o "computational thinking" fue introducido por vez primera dentro de la comunidad científica en computación, a través de un artículo de ACM Communications escrito por Jeannette Wing en 2006, profesora en Carnegie Mellon University en Pittsburg, Estados Unidos y al mismo tiempo presidenta del Departamento de Ciencias Computacionales del mismo instituto. Es la mencionada autora quien formula la definición del término como una forma de pensar que no se restringe en exclusiva para programadores de sistemas ni científicos en computación, sino como habilidades útiles para todo el mundo.

En este sentido la autora expresa que el pensamiento computacional "consiste en la resolución de problemas, en el diseño de los sistemas y en la comprensión de la conducta humana haciendo uso de los conceptos fundamentales de la ciencia informática" (Wings, 2006, p. 33). Esta definición incluye a su vez un considerable rango de herramientas mentales que reflejan y ponen de manifiesto la amplitud del campo potencial individual.

En correspondencia con el origen del término, existen teóricos que otorgan crédito al matemático Seymour Papert por ser el primero en aproximar una definición de pensamiento computacional. Papert es cofundador del Laboratorio de Inteligencia Artificial del Instituto Tecnológico de Massachusetts (MIT), e incluso, frecuentemente se relata que los primeros juguetes para niños con computación incorporada fueron creados en el interior de su laboratorio durante la década de los años sesenta

Por su parte, Repenning y Lamprou (2016) comentan que existen tres escenarios primordiales capaces de describir la relación entre las responsabilidades humanas $y$ computarizadas, asimismo fundamentan sus argumentos desde las concepciones de Wing sobre el pensamiento computacional y lo segmentan en tres partes que a continuación se señalan:

- Formulación del problema (proceso de abstracción): abstraer significa extraer la idea general de un todo, dentro de este proceso mental es necesario que la persona exprese la formulación de una interrogante en torno a los sucesos a analizar: ¿cómo algo funciona?; igualmente puede visualizar un determinado problema usando un diagrama sobre un trozo de papel, por ejemplo. Dicho esto, es claro que en este apartado no es necesario el uso del computador para inicializar las labores de un pensador computacional.

- Expresión de soluciones (automatización): una forma de llegar a las soluciones es manejar expresiones no ambiguas respecto a las mismas, para que la computadora pueda llevarlo a 
cabo mediante los procesos aplicados en programación computacional.

- Ejecución y Evaluación (análisis): la ejecución de soluciones por el computador muestra consecuencias directas sobre la propia forma de percibir el mundo, debido a que el pensamiento computacional es una destreza importante, la educación en general y la formación en las ciencias informáticas particularmente, deben mantener el foco sobre la creación de oportunidades para nuevos pensadores computacionales y no exclusivamente para programadores de software.

Otros expertos en el área establecen que el pensamiento computacional es un enfoque de solución de casos que empodera la integración de tecnologías digitales conjuntamente con las ideas humanas, en el que no se reemplaza el énfasis en la creatividad, razonamiento $\mathrm{o}$ pensamiento crítico, pero refuerza esas habilidades al tiempo que realza formas alternativas para organizar el problema de manera que el computador pueda ayudar en la labor Sociedad Internacional para la Tecnología en Educación (ISTE) y Asociación de Docentes en Ciencias de la Computación (CSTA), (2011).

Siguiendo el orden documental, el equipo que desarrolla el software de programación scratch, quienes son mundialmente reconocidos por sus aportes al estudio de la lógica dentro de las aplicaciones informáticas del MIT, definen el pensamiento computacional como un conjunto de conceptos, prácticas y perspectivas basadas en las precisiones del mundo de la informática.

En este sentido, los investigadores recalcan que los estudiantes al programar y compartir proyectos, comienzan a desarrollarse como pensadores computacionales, aprendiendo nociones y configuraciones básicas de computación y matemáticas, a la vez que practican estrategias para el diseño, resolución de problemas y otras formas de colaboración entre pares (ScratchEd Team, 2015).

En resumen, puede decirse que esta clase de pensamiento, el pensamiento computacional, no debe confundirse con la codificación de sistemas lógicos. Este es un tipo de pensamiento beneficioso para usuarios que no efectúan operaciones mediante el computador.

En efecto, el pensamiento computacional es un método estructurado, comprobado y diseñado para identificar problemas; éste tiene lugar desde la óptica individual en la que se percibe el mundo y sus eventualidades, guardando vínculo con las técnicas para la programación mental, y los hábitos y entrenamientos del músculo cerebral, sin distinciones de edad o nivel de alfabetización informática que tenga el interesado.

Es así como este tipo de pensamiento se compone en cuatro partes primarias que se pueden observar en la Figura 1, las cuales incluyen la descomposición (desglosar un problema complejo o sistema en partes más pequeñas y manejables), el reconocimiento de patrones (buscar similitudes dentro de los problemas presentados), la abstracción o generalización del patrón (centrarse 
exclusivamente en la información importante, ignorando los diseños irrelevantes de detalles) y el diseño de algoritmos (desarrollo de una solución paso a paso para el problema, o las reglas a seguir para resolver algo), frecuentemente en este marco se asocia una etapa final, la cual se corresponde con la depuración o "evaluación" de los resultados, refiriéndose al perfeccionamiento de los anteriores pasos (Cummins, 2016).

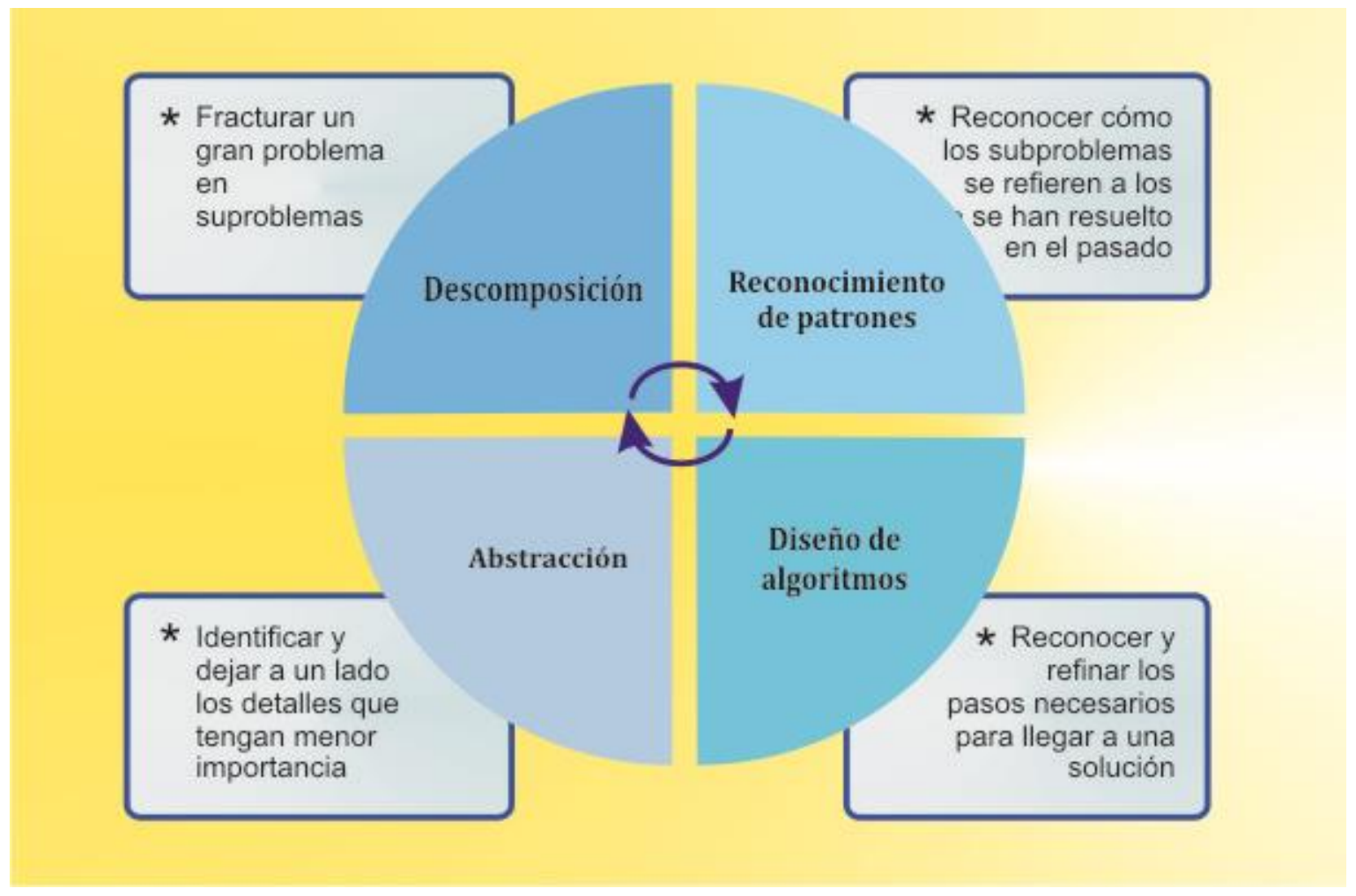

Figura 1. Etapas del pensamiento computacional.

\section{Importancia del pensamiento computacional}

Naturalmente, las variadas disciplinas del saber universal continúan expandiéndose con el paso del tiempo y los cambios en las realidades, desde la indudable interconexión entre las ciencias, la mezcla no integrativa de campos que implican la multidisciplinariedad, y en la forma en que cada día se obtienen nuevas y profundas compresiones en cada ámbito funcional.
En esencia, la pertinencia de activar diferentes estilos de pensamiento se origina desde la existencia de problemas científicos que siguen siendo intelectualmente desafiantes y atractivos para una comunidad de analistas que desean trabajar en la pesquisa y resolución de solicitudes concretamente presenciadas en el medio, teniendo en unión un espacio problemático acompañado por un entorno de solución, que se condensa a través de dos 
importantes cualidades humanas: la curiosidad dada en toda circunstancia y la creatividad con la que se aborden las trabas.

Los individuos pueden especializarse en ciencias de la computación y desempeñar cualquier rol en el sector laboral, igualmente una persona puede especializarse en inglés o matemáticas y pasar a una multitud de carreras diferentes (Wing, 2009). Para la autora, alguien puede especializarse en ciencias de la computación e ir a una carrera en medicina, derecho, negocios, política, así como a cualquier ciencia o ingeniería, e incluso incorporar sus esfuerzos para las artes; más obviamente, el ambiente interdisciplinario de las ciencias informáticas significa especializarse en procesos $y$ organización de computadores, permitiendo a un estudiante emprender una carrera relacionada con una disciplina alternativa o en los límites de muchos, esto se logra pensando estructuradamente.

Al estudiar ciencias de la computación, un aprendiz se empodera de una forma específica de pensar (Wing, 2006) y es este punto el que marca el innegable valor de poder pensar computacionalmente. Este hecho ha sido considerado en muchas regiones del mundo, donde planifican lecciones para afinar el desarrollo de procesos lógicos con lenguajes de programación de nivel básico como Scratch o Kodu Game Lab, así como el software Alice, el Entorno de Desarrollo Online Codio u otras herramientas.

De igual manera, el énfasis de esta investigación estuvo dada en función de la oportunidad que ofrece de activar diferentes formas de pensar a jóvenes aprendices, tomando como modelo el currículo nacional de Inglaterra, donde se establece que a los niños se les debe ofrecer la educación de computación de alta calidad, para justamente, trabajar los componentes de este pensamiento, como habilidades únicas en el entendimiento y transformación mundial (Berry, 2013); mientras que, al observar más allá de la educación, importantes empresas como Google y Microsoft Research, se puede evidenciar que consideran al pensamiento computacional como un conjunto esencial de experiencias y métodos de resolución de problemas para los ingenieros de software.

En este mismo orden de ideas y referido a la importancia de este estilo de pensamiento, Bourn (2018) indica que el pensamiento computacional es una habilidad que debe ser enseñada a los niños, para garantizar que puedan estar listos para el lugar de trabajo que luego asumirán y logren participar eficazmente dentro de este medio digital. Así en esta acotación del autor se expresa que las habilidades inherentes a este método son beneficiosas prácticamente para las carreras de todas las áreas, incluidos los productos de consumo, mercados empresariales y financieros, la energía, los viajes y el turismo, o los servicios públicos como la salud, la educación, la ley y las propias regulaciones de cualquier país.

Los lugares de trabajo necesitan que los empleados tomen un papel más activo en el procesamiento de los problemas a través de sus pensamientos, en función de la creación de soluciones.

Desde una mirada educativa, para aprovechar el gradual potencial del 
pensamiento computacional las escuelas deberían abordar tres desafíos fundamentales: en primer lugar, los profesores necesitan ser adecuadamente capacitados y apoyados para enseñar el currículo de computación con confianza (en ocasiones se observa una realidad donde los profesores a cargo de materias como computación o programación no son especialistas en el área).

En segundo lugar, los directores y líderes curriculares deberían estar financiando la computación adecuadamente y brindándole la importancia que merece (algunas escuelas todavía no enseñan computación como una asignatura independiente, sino que la incorporan en otras materias).

Por último, las escuelas deben defender la teoría y práctica en conjunto, porque estos dos factores no son mutuamente excluyentes $y$, cuando se combinan, pueden conducir a resultados fantásticos en las aulas y producir jóvenes con una gran habilidad para asumir el lugar de trabajo (BCS The Chartered Institute for IT, 2015).

En cuanto a las habilidades específicas del pensamiento computacional, diversas han sido las aproximaciones a las virtudes y potentes atributos que puede ganar un pensador computacional dentro de este trabajo, desde la articulación de elementos mentales para la generación de ideas, hasta la aplicación de resoluciones que pueden ser efectivamente aplicadas en la cotidianidad de cualquier contexto. Sin embargo, es momento de hacer énfasis en una destreza particular, que se relaciona con la producción de sistemas, pero que se pretende proclamar como opción práctica para la vida, ésta a saber es, la competencia de codificar.

Efectivamente, escribir códigos muestra una parte más visible de la manera en la que se piensa, que es válida no sólo en ese ámbito de la actividad mental que sostiene el desarrollo y la creación de programas o sistemas; hay una forma específica de deliberar, de organizar ideas y representaciones sobre algo que desde luego favorece las competencias computacionales.

Al hacer mención a los procesos de codificación, se alude a una forma de pensar propicia para el análisis y la relación de ideas, para la organización y representación lógica, esas habilidades se ven favorecidas con ciertas actividades y entornos concretos de aprendizaje desde las primeras etapas. Se trata del desarrollo de un pensamiento específico, de un pensamiento computacional (Zapata-Ros, 2015). Consecutivamente, en la Tabla 1 se resumen las habilidades del pensamiento computacional, según Sharples et al. (2016), quienes extractan este concepto, no sólo como una manera de pensar, sino también una forma de trabajar en los problemas. 
Tabla 1. Habilidades del pensamiento computacional

\begin{tabular}{lll}
\hline \multicolumn{1}{c}{ Habilidad } & Descripción \\
\hline 1 & Pruebas y depuración. & $\begin{array}{l}\text { Encontrar y resolver los problemas a medida } \\
\text { que se trabajan. }\end{array}$ \\
2 & Reutilización y remezcla. & Producir sobre proyectos o ideas existentes. \\
3 & Abstracción y modularización. & $\begin{array}{l}\text { Explorar las conexiones entre el todo y las } \\
\text { partes que lo constituyen. }\end{array}$ \\
5 & Expresión. & $\begin{array}{l}\text { Reconociendo que de esta manera, el trabajo } \\
\text { es una actividad creativa. }\end{array}$ \\
& Conexión. & $\begin{array}{l}\text { Reconociendo el poder de crear con y para los } \\
\text { demás. }\end{array}$ \\
& Cuestionamiento. & $\begin{array}{l}\text { Sentirse facultado para hacer preguntas sobre } \\
\text { el mundo. }\end{array}$
\end{tabular}

Fuente: Sharples et al. (2016)

La definición operativa de ISTE (2011) sobre el pensamiento computacional para la educación arroja más luz sobre las habilidades que este soporta, incluyendo: confianza en el tratamiento de la complejidad, perención en el trabajo con problemas difíciles, tolerancia para la ambigüedad, capacidad de lidiar con problemas de extremo abierto, capacidad de comunicarse y trabajar con otros para lograr un objetivo común, lo que los autores consideran no son sólo atributos de un programador fuerte, sino que son competencias de un empleado fuerte.

Estos resultados ponen de relieve que el pensamiento computacional es más que un conjunto de medidas para ser trabajadas, el adjetivo "computacional" no implica netamente que a los seres humanos se les enseñe a pensar como máquinas sin imaginación, que mayormente sólo pueden resolver un problema cuando éste se suministra con un conjunto preparado de instrucciones a seguir; el pensamiento computacional es una forma en la que los seres humanos pueden pensar al momento de estar inmersos en las intenciones para la resolución de problemas (Sharples et al., 2016).

Como enfoque el pensamiento computacional se puede configurar como un conjunto de medidas claras, pero la separación y la navegación por estos pasos es una actividad humana creativa, por tanto, Zapata-Ros (2015) coincide plenamente en que el pensamiento computacional va más allá de saber usar dispositivos digitales, de codificar, programar o aplicar pensamiento lógico matemático, aun cuando este último es su base y los primeros sus manifestaciones más comunes.

En efecto, además de lo antes mencionado el pensamiento computacional incluye otros dominios como la teoría 
general de sistemas, gran organizadora en la observación del mundo y la sistematización como gran organizadora del hacer, la inteligencia artificial como aproximación a la comprensión de la inteligencia, los modelos computacionales y simulaciones como formas de experimentación en las ciencias y demostración en las matemáticas, la ubicuidad de las tecnologías de información y comunicación (TIC), entre otros aspectos que hacen probable que el futuro cercano incorpore los conceptos tanto de mente extendida y compartida como de inteligencia colectiva.

Paralelamente, Rojas (2015) expone en sus estudios las numerosas bondades que posee el pensamiento lógico en los niños, como un elemento crucial en el cuerpo curricular de la infancia, por lo que plantear estrategias que potencien el pensamiento lógico en las aulas, se convierte en una necesidad apremiante, a la vez que se constituye en uno de los principales retos para la comunidad de maestros, no siendo exclusivo de la rama de las matemáticas sino que es transversal a las demás disciplinas.

En este sentido, el citado autor manifiesta como ejemplo que para adquirir la habilidad de contar, se requiere que el niño asemeje diversos principios lógicos, entre los que se destacan la comprensión de la naturaleza del orden de los números (sin olvidar el concepto de magnitud), entendimiento del concepto de unicidad en el orden; cada número debe contarse una única vez, y la asimilación de que el número final de una serie representa la totalidad de elementos de una colección.
En este mismo orden de ideas, es necesario resaltar que, cuando se piensa en hacer que un infante ordene sus juguetes, escriba sus rituales matutinos como una secuencia de pasos, o seguir una receta al hornear galletas, por ejemplo, se le enseña la relación entre un objetivo deseado y la secuencia correspondiente de pasos a ejecutarse, tal como deben hacerlo al escribir códigos.

De igual forma, para los casos de niños con dificultades de atención, hiperactividad o aquellos a los que no les gusta leer, escribir o calcular, este tipo de pensamiento les permite manejar instrucciones, concentrarse en ideas claves, buscar patrones y divertirse, a la vez que afianzan sus potencialidades.

\section{MÉTODO}

La investigación se enmarcó en una metodología con diseño documental bibliográfico. La planeación del trabajo se constituyó en pasos secuenciales que iniciaron con la selección y delimitación del tema en cuestión, recolección de información necesaria por medio de un arqueo general, desde la consulta a diversas fuentes bibliográficas impresas y electrónicas, seguidamente se procedió a la organización, análisis e interpretación de la información recabada. Por último, se presentan dentro de la redacción del documento los resultados obtenidos a través del procesamiento y comprensión de los textos a fin de lograr una reflexión y postura crítica ante la temática que se estudió. 
RESULTADOS

En virtud de administrar un sistema para el reforzamiento de las habilidades intelectuales de los niños y teniendo en consideración las teorías sobre la existencia de inteligencias múltiples en el ser humano; los docentes, representantes y comunidad educativa en general, deben ser esencialmente sensibles ante la manifiesta necesidad de brindar soporte a determinados quehaceres, que jueguen un rol estimulante en la actividad cerebral de los jóvenes aprendices, siendo conscientes además, que tal entrenamiento debe cumplir con algunos atributos, como la progresividad (mantenimiento de continuos ritmos en las actividades e incluir, a medida que se va avanzando en los contenidos, nuevos niveles de complejidad en las dinámicas que se propongan) y el entretenimiento.

Como consecuencia de lo antes expuesto, se planteó por un lado, que las actividades desarrolladas en el marco de la instrucción formal o informal, sean rediseñadas en orden de acatar los elementos y etapas que caracterizan al pensamiento computacional. De esta manera se puede apostar a la consolidación de aprendizajes fundamentados en la aprehensión de esquemas mentales superiores, para la resolución de cuestiones académicas y cotidianas.

En efecto, el rediseño de actividades mediante la coordinación de estrategias útiles para pensar creativamente, inspirar a niños y jóvenes a mantenerse curiosos ante el mundo, crear nuevos conceptos $y$, por encima de todos estos aspectos, activar ambientes divertidos y motivadores para la mediación de aprendizajes, es una necesidad para establecer el pensamiento computacional como una competencia primordial en el desarrollo cognitivo de los estudiantes.

Para el presente caso de investigación, se sintetizan una serie de estrategias potenciales que pueden ser configuradas de acuerdo a la etapa evolutiva de los estudiantes. Estas actividades fueron diseñadas para grupos de niños en etapa escolar primaria, para lo cual se estableció un rango de entre los 6 a 11 años de edad. Sin embargo, las temáticas y directrices pueden ser adaptadas oportunamente, en función a las competencias que se aspiren desarrollar en el estudiantado.

Asimismo, de manera general se seleccionó como temática común para ejemplificar las estrategias, algunos contenidos sobre astronomía básica y las generalidades del sistema solar, por ser un aspecto de interés global en niños y que en este caso fue denominado "campamento espacial", un compendio de actividades con temática de planetas, que tuvo como principal objetivo el desarrollo de habilidades intelectuales, desde la perspectiva que suscita cada etapa del pensamiento computacional (ver Tabla 2). De igual manera cada modelo presentado en este caso, puede ser modificado y personalizado para cumplir los objetivos de aprendizaje de cada diseño curricular o plan de clases, según la temática requerida y la edad de los grupos. 
Tabla 2. Estrategias propuestas en el "campamento espacial" para estimular el pensamiento computacional en estudiantes de educación básica

\begin{tabular}{ccc}
\hline $\begin{array}{c}\text { Etapa del } \\
\text { pensamiento } \\
\text { computacional }\end{array}$ & Nombre y descripción de la estrategia & Habilidades a desarrollar \\
\hline
\end{tabular}

1 Descomposición.

2 Abstracción.
Tu propio plano de un cohete: la actividad consiste en dibujar un plano descriptivo con las partes de un cohete espacial. Es notoria la enorme complejidad que puede tener esta tarea, sin embargo, en una hoja de papel, el niño debe fragmentar en cuadros de igual dimensión, el espacio en el que hará el diseño. Luego, teniendo una fotografía de referencia, puede ir dibujando cuadro a cuadro, las partes de la nave, para facilitar su realización. A su vez, puede descomponer el cohete según sus partes, haciendo una lista de las piezas, describir cada módulo del cohete y juntarlas imaginariamente para ratificar que nada resta por colocar en el plano.

Ficha de planetas del Sistema Solar: en esta actividad, el niño debe leer los datos y razonar acerca de los enunciados presentados:

- Mercurio es un planeta, gira alrededor del Sol, su traslación es de 88 días. Es el más pequeño.

- Venus es un planeta, gira alrededor del Sol, su traslación es de 225 días.

- Tierra es un planeta, gira alrededor del Sol, su traslación es de 365 días.

- Marte es un planeta, gira alrededor del Sol, su traslación es de 1,9 años.

- Júpiter es un planeta, gira alrededor del Sol, su traslación es de 11,9 años. Es el más grande.

- Saturno es un planeta, gira alrededor del Sol, su traslación es de 29,7 años.

- Urano es un planeta, gira alrededor del Sol, su traslación es de 83,7 años.

- Neptuno es un planeta, gira alrededor del Sol, su traslación es de 166 años (El Nacional, 2004).

Posteriormente, el niño deberá organizar la información en síntesis, siguiendo una estructura similar a esta:

1. Mercurio, Venus, Tierra, Marte, Júpiter, Saturno, Urano y Neptuno son planetas.

2. X planeta gira alrededor del Sol.

3. $\mathbf{X}$ planeta tiene $\mathbf{X}$ cantidad de tiempo de traslación.

4. Mercurio es más pequeño que 7 planetas.

5. Júpiter es más grande que 7 planetas.

Nota: se pueden elaborar tantas interrogantes como sean posibles, con las que el estudiante pueda asociar, comparar, ampliar el contenido y abstraer ideas generales sobre el mismo.
El niño logra romper una tarea que puede resultarle compleja de realizar al inicio, esto implica dividir en módulos el problema, para abordarlo en escalas menos exigentes, luego unifica todo el procedimiento y llega a la solución igualmente.

Se desarrollan las habilidades de: reutilización y remezcla, modularización, expresión.

El niño puede interpretar los conceptos en función de llegar a conclusiones generalizadas. Por un lado, logra ignorar ciertos detalles de lo estudiado para concentrarse en las ideas principales. De la misma manera, es capaz de extraer lo esencial, partiendo de un todo. Se desarrollan las habilidades de: abstracción modularización, cuestionamiento. 


\begin{tabular}{ccc}
\hline $\begin{array}{c}\text { Etapa del } \\
\text { pensamiento } \\
\text { computacional }\end{array}$ & Nombre y descripción de la estrategia & $\begin{array}{c}\text { Habilidades a } \\
\text { desarrollar }\end{array}$ \\
\hline
\end{tabular}

3 Reconocimiento de patrones.
4 Diseño algoritmos.
¿Qué llevar en la mochila?: se efectúa una lectura a los niños donde se expliquen los movimientos de la Tierra en su órbita alrededor del Sol. Se indica que durante la traslación, el planeta pasa por posiciones: los solsticios de verano e invierno y los equinoccios de primavera y otoño. Se continúa el debate mientras se formula una pregunta:

"¿Qué objetos llevarías en tu mochila durante el verano? y ¿cómo vestirías?"

La pregunta se presentaría de la misma manera para las cuatro estaciones y cada niño puede "preparar" su equipaje de acuerdo a cada temporada. Puede realizarse verbalmente, a través de dibujos, fichas o rompecabezas; con los que los niños puedan imaginar el ambiente.
Para el caso de la actividad de la mochila, el niño conoce qué tipo de vestimenta debe portar según la estación, porque existen patrones en su vida que orientan sus disposiciones actuales. La dinámica le permite identificar patrones, partiendo de lo que conoce. De acuerdo a los estándares observados o vividos, el niño sabe cómo condicionar comportamientos $y$ decisiones, así como también, entiende diversas cuestiones del entorno (sabe de qué forma obtener lo que espera).

Se desarrolla la
habilidad de
cuestionamiento.
cuestionamiento. El niño es capaz de ordenar una serie de instrucciones, que sean fáciles de seguir por otros y que impliquen la solución de algo.

Mediante el pensamiento algorítmico, el aprendiz aplica lógica y ordenamiento secuencial para trabajar en sus tareas.

Se desarrollan las habilidades de: reutilización y remezcla, conexión. 


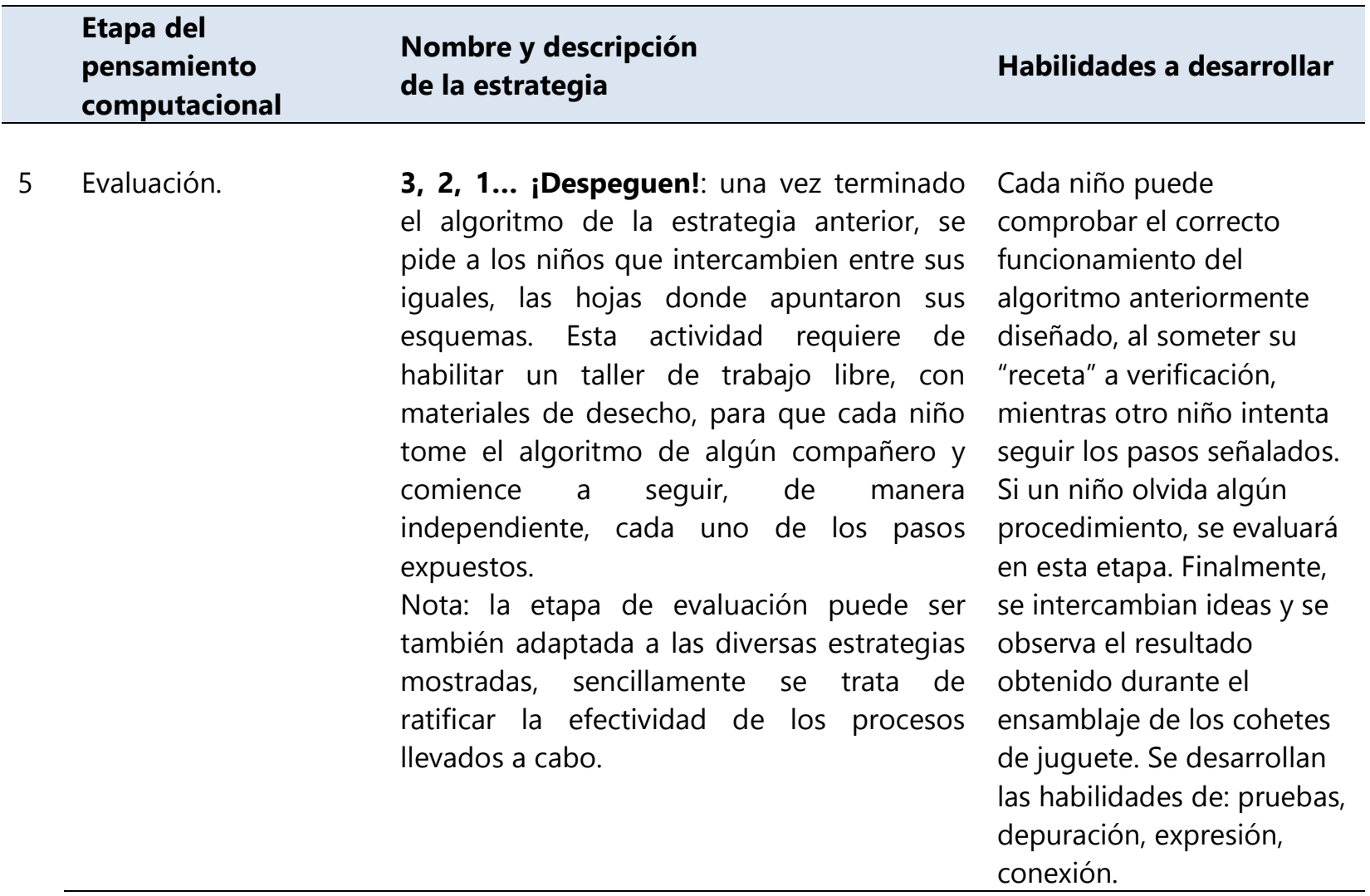

El aspecto positivo en torno a estas actividades, es que requieren pocos recursos para ser manejadas en el aula o en el hogar. Para la activación de estas estrategias pueden confeccionarse fichas, tarjetas, representaciones gráficas, anotaciones en los cuadernos de apuntes, dibujos en el pizarrón, elaboración de diseños digitales, presentaciones con diapositivas o cualquier medio instruccional que permita el apoyo, tangible o intangible.

De igual forma las actividades pueden desarrollarse haciendo uso de la imaginación, a través de lecturas guiadas, uso de material de reciclaje, mímicas, cuentos, conversaciones, e incluso, dibujos en la arena en espacios abiertos. El ideal, es aminorar en lo posible los recursos económicos que se pretendan disponer para su adecuación, debido a que, como se señalaba, se busca incorporar estas u otras estrategias a la planificación de clases, de manera permanente.

\section{CONCLUSIONES}

Finalmente, el cerebro humano debe ser alimentado a cada instante por el entorno y por el individuo mismo, es por ello que, como lo indican diversas teorías, este órgano busca estar siempre en confort, procura crear paz ante el caos y trata de organizar los estímulos capturados por otros componentes del cuerpo.

Por naturaleza, el cerebro humano intenta con frecuencia obtener placer, de allí que resulte lógico concluir que, posiblemente un método de aprovechamiento ante esta cualidad, reside en la construcción de una adecuada programación neurológica, compendiada en 
la creación de hábitos de estudio, en el disfrute de una inmensa curiosidad ante diversas interrogantes de vida, en la aplicación de la creatividad, búsqueda de inspiración, y en pensar de manera divergente; de modo que se puedan forjar en el cerebro patrones de costumbre en los que el placer se encuentre relacionado con procesos del intelecto.

En pocas palabras, tal como lo hacen los sistemas lógicos, si un humano se enfoca en funcionar, mediante la lectura de entradas (instrucciones específicas) basadas en un lenguaje oriundo para sí (que adicionalmente puedan ser -previa y eficientemente- programadas), entonces el seguimiento de un "script" o guión interno, podría configurar sus formas de pensar y resolver conflictos, intensificando las oportunidades para alcanzar verdaderas proezas individuales.

En estas líneas finales se afirma la relevancia de insistir en la activación de nuevos retos para abordar el aprendizaje, activación basada en la capacidad que tengan los individuos para generar nuevos $y$ propios conocimientos así como la capacidad de descubrir sus potencialidades.

Específicamente en educación, el pensamiento computacional es importante para el desarrollo integral del estudiante, no tratándose precisamente de que éste comprenda los fundamentos para la programación de software, códigos o lenguajes, tampoco temas sobre computación en sí mismos; sino que, el término sugiere la aplicación de técnicas para aprender a pensar ordenadamente, siguiendo patrones de inmediatez y eficiencia en lo que se hace.
En esencia, esta revisión teórica expone la percepción del pensamiento individual, y parte del entendimiento de la forma en la que funciona la tecnología digital y aplica métodos para afrontar cuestiones mentales que apunten a la satisfacción de necesidades, sea, al descomponer un todo en sus partes, o bien cuando asocia unos elementos con otros previamente revisados, reconoce patrones en serie, compara y revisa información en todo momento, siente curiosidad ante determinados eventos, aplica creatividad, entre otros procedimientos. El pensamiento computacional, concretamente sirve como herramienta para reconstruir los métodos de pensamiento, aprender haciendo, manejar ideas, proponer reformas, proyectos o creaciones que sean adaptables a los intereses sociales.

\section{REFERENCIAS}

BCS The Chartered Institute for IT (2015). The benefits of computational thinking. Recuperado de https://www.bcs.org/content-hub/thebenefits-of-computational-thinking/

Berry, M. (2013). Computing in the National Curriculum: a guide for primary teachers. Bedford, UK: Computing at School. Recuperado de http://www.computingatschool.org.uk/da ta/uploads/CASPrimaryComputing.pdf

Bourn, C. (2018). What is computational thinking, and why should all kids learn it? Primo Toys. Recuperado de https://www. primotoys.com/what-iscomputational-thinking-and-why-care/

Cummins, K. (2016). Five reasons why computational thinking is an essential tool for teachers and students. Edgalaxy.com - Cool stuff for nerdy teachers. Recuperado de https://www.edgalaxy.com/journal/2016/ 
5/25/five-reasons-why-computationalthinking-is-an-essential-tool-forteachers-and-students

International Society for Technology in Education (ISTE) (2011). Computational Thinking Leadership Toolkit, first edition. Recuperado de http://www.iste.org/docs/ctdocuments/ct-leadershipttoolkit.pdf?sfvrsn=4

International Society for Technology in Education (ISTE) and Computer Science Teachers Association (CSTA) (2011). Pensamiento computacional: recursos para Profesores. Recuperado de http://csta.acm.org/Curriculum/sub/CurrF iles/472.11CTTeacherResources_2ed-SPvF.pdf

Repenning, A. y Lamprou, A. (2016). Computational Thinking $\neq$ Programming. SI Digital Magazine-Schweizer Informatik Gesellschaft. Recuperado de https://magazine.swissinformatics.org/en /computational-thinking-\%E2\%89\%A0programming/

Rojas, S. (2015). La importancia del pensamiento lógico. Redacción El Educador. Recuperado de http://www.eleducador.com/laimportancia-del-pensamiento-logico/
Sharples, M., Adams, A., Alozie, N., Ferguson, R., FitzGerald, E., Gaved, M., McAndrew, P., Means, B., Remold, J., Rienties, B., Roschelle, J., Vogt, K., Whitelock, D. y Yarnall, L. (2016). Innovating Pedagogy: Open University Innovation Report 4. Milton Keynes: The Open University. Recuperado de http://innovatingpedagogy.wikidot.com/pensamientocomputacional

Wing, J. (2006). Computational Thinking. Communications of the ACM, 49(3), 3335. Recuperado de https://www.cs.cmu.edu/ 15110s13/Wing06-ct.pdf

Wing, J. (2009). Computational Thinking and thinking about computing. OpenLearn, The Open University. Recuperado de https://www.open.edu/openlearn/science -maths-technology/computing-andict/introduction-computationalthinking/content-section-0?intro $=1$

Zapata-Ros, M. (2015). Pensamiento computacional: una nueva alfabetización digital - Computational thinking a new digital literacy. RED: Revista de Educación a Distancia. 46(4). Recuperado de https://www.um.es/ead/red/46/zapata.pdf 